\title{
Reconstruction of Dynamic PET Data Using Spatio-Temporal Wavelet $\ell_{1}$ Regularization
}

\author{
Jeroen Verhaeghe, Dimitri Van De Ville, Ildar Khalidov, Michael Unser, Yves D’Asseler and Ignace Lemahieu
}

\begin{abstract}
Tomographic reconstruction from PET data is an ill-posed problem that requires regularization. Recently, Daubechies et al. [1] proposed an $\ell_{1}$ regularization of the wavelet coefficients that can be optimized using iterative thresholding schemes. In this paper, we extend this approach for the reconstruction of dynamic (spatio-temporal) PET data. Instead of using classical wavelets in the temporal dimension, we introduce exponential-spline wavelets that are specially tailored to model time activity curves (TACs) in PET. We show the usefulness of spatio-temporal regularization and the superior performance of E-spline wavelets over conventional BattleLemarié wavelets for a 1-D TAC fitting experiment and a tomographic reconstruction experiment.
\end{abstract}

\section{INTRODUCTION}

Dynamic Positron Emission Tomography (PET) reconstruction is challenging due to the small number of accumulated counts in each time-bin. One popular approach is to use a regularization term, which acts as an implicit image model, to make the inverse problem well-conditioned [2]. In [1], the authors proposed a sparsity constraint by an $\ell_{1}$ norm on the spatial wavelet coefficients $\boldsymbol{w}$, which leads to the criterion

$$
\lambda\left(x_{1}, x_{2}\right)=\arg \min _{\lambda \geq 0}\left\{\|\boldsymbol{y}-\mathrm{P} \lambda\|_{2}^{2}+\mu\|\boldsymbol{w}\|_{1}\right\},
$$

where the imaging operator $\mathrm{P}$ expresses the forward model from the spatial object $\lambda\left(x_{1}, x_{2}\right)$ to the observations $\boldsymbol{y}$, and where $\mu$ is the tuning parameter. The image $\lambda\left(x_{1}, x_{2}\right)$ is expanded on an orthogonal wavelet basis with wavelet coefficients $\boldsymbol{w}$, the $\ell_{1}$-term favors a sparse representation. Most interestingly, this criterion can be optimized using an iterative thresholding algorithm.

In this paper, we extend the non-parametric reconstruction that follows from (1) for the reconstruction of dynamic (spatio-temporal) PET data. Instead of using conventional wavelets in the temporal dimension, we opt for exponentialspline wavelets [3] that are tuned to represent well TACs as encounted in dynamic PET imaging. The key concept is that the activity distribution in the body is ruled by systems of differential equations involving so-called compartmental models. The proposed E-spline wavelets are ideally suited for the sparse representation of solutions of these differential equations.

This work was supported by BOF and FWO

J. Verhaeghe, Y. D'Asseler, I. Lemahieu are with ELIS-MEDISIP, Ghent University, Sint Pietersnieuwstraat 41, 9000 Ghent, Belgium

D. Van De Ville, I. Khalidov and M. Unser are with BIG, EPFL, Lausanne, Switzerland

E-mail: jeroen.verhaeghe@ugent.be

\section{MATERIALS AND METHODS}

\section{A. Problem formulation}

We consider the problem of reconstructing a (nonnegative) spatio-temporal activity distribution $\lambda\left(x_{1}, x_{2}, \tau\right)$ from dynamic PET data $\boldsymbol{y}=\mathrm{P} \lambda$ [2]. The reconstruction task is to find the non-negative minimizer of the criterion

$$
\left\|\boldsymbol{y}-\mathrm{P}\left\{\lambda\left(x_{1}, x_{2}, \tau\right)\right\}\right\|_{2}^{2}+\mu\|\boldsymbol{w}\|_{1} .
$$

The choice of the spatio-temporal wavelet decomposition that determines the coefficients $\boldsymbol{w}$ is explained next.

\section{B. Wavelet Representation}

We represent the activity distribution $\lambda\left(x_{1}, x_{2}, \tau\right)$ on an orthogonal wavelet basis [4], [5].

$$
\lambda\left(x_{1}, x_{2}, \tau\right)=\sum_{\boldsymbol{i}} \sum_{\boldsymbol{k}} w_{\boldsymbol{j}}[\boldsymbol{k}] \psi_{\boldsymbol{j}, \boldsymbol{k}}\left(x_{1}, x_{2}, \tau\right),
$$

with $i$ and $\boldsymbol{k}$ are the scale and translation parameters. We use wavelets that are separable in space and time:

$$
\psi_{\boldsymbol{j}, \boldsymbol{k}}\left(x_{1}, x_{2}, \tau\right)=\psi_{k_{1}, j_{1}}^{(1)}\left(x_{1}\right) \psi_{k_{2}, j_{2}}^{(1)}\left(x_{2}\right) \psi_{k_{3}, j_{3}}^{(2)}(\tau) .
$$

We include the (coarest-scale) scaling functions in this notation which by convention crorrespond to $j=j^{\max }$. We consider different decomposition levels in the spatial and temporal domain, i.e. $j_{1}^{\max }=j_{2}^{\max }=J_{s}$ and $j_{3}^{\max }=J_{t}$.

In this work, $\psi^{(1)}(x)$ are polynomial B-spline wavelets [5], $\psi^{(2)}(\tau)$ are E-spline wavelets [3]. We will use here Esplines wavelets, as they possess some interesting properties that are presented in the next section.

\section{Exponential spline wavelets}

A key property of the B-spline wavelets is that they have $N$ vanishing moments, where $N$ is the order of the scaling function. The E-spline wavelets, on the other hand have $N$ vanishing exponential moments [3], [6], [7]; i.e. we have that

$$
\int_{-\infty}^{+\infty} p_{\boldsymbol{\alpha}}(\tau) \psi_{j}^{(2) *}\left(\tau-\tau_{0}\right) d \tau=0
$$

where $\boldsymbol{\alpha}$ is the E-spline-defining parameter vector and $p_{\boldsymbol{\alpha}}(\tau)$ is the corresponding exponential polynomial given by:

$$
p_{\boldsymbol{\alpha}}(\tau)=\sum_{k=1}^{N_{d}} \sum_{n=0}^{m_{k}-1} c_{k} \tau^{n} e^{\alpha_{k} \tau},
$$

$\boldsymbol{\alpha}$ consists of $N_{d}$ distinct $\alpha_{k}$ 's of multiplicity $\left\{m_{k}\right\}_{1}^{N_{d}}$ with $N=\sum_{k}^{N_{d}} m_{k}$. 
The wavelets are closely related to the linear differential system $L\{y\}=x$ whose transfer function in the Laplace domain is given by

$$
L_{\boldsymbol{\alpha}, \boldsymbol{\gamma}}(s)=\frac{\prod_{n=1}^{N}\left(s-\alpha_{n}\right)}{\prod_{n=1}^{M}\left(s-\gamma_{m}\right)} .
$$

Indeed, it can be seen that the exponential polynomials $p_{\boldsymbol{\alpha}}$ are in the null space of the operator $L_{\boldsymbol{\alpha}, \boldsymbol{\gamma}}$.

\section{The iterative thresholding algorithm}

To find the minimizer of (2) we use the same iteration scheme as proposed in [1], which computes the next estimate as

$$
\lambda^{n+1}=\mathcal{P} S_{\mu}\left(\lambda^{n}+\mathrm{P}^{T}\left(y-\mathrm{P} \lambda^{n}\right)\right), \quad n=0,1, \ldots,
$$

where $\mathcal{P}$ is the projector on the non-negative functions and the non-linear operator $S_{\mu}$ is defined componentwise by

$$
\mathrm{S}_{\mu}(g)=\sum_{j, k} \mathcal{S}_{\mu}\left(w_{j}[k]\right) \psi_{j, k}=\sum_{j, k} \mathcal{S}_{\mu}\left(\left\langle g, \psi_{j, k}\right\rangle\right) \psi_{j, k}
$$

where $\mathcal{S}_{\mu}$ is the soft-thresholding function

$$
\mathcal{S}_{\mu}(x)= \begin{cases}x+\frac{\mu}{2} & \text { if } x \leq-\frac{\mu}{2} \\ 0 & \text { if }|x|<0 \\ x-\frac{\mu}{2} & \text { if } x \geq \frac{\mu}{2}\end{cases}
$$

\section{E. Implementation}

The analysis and synthesis steps can be implemented by means of a filter bank [4]. Explicit expressions for designing the E-spline wavelet filters for given $\alpha$ and $\gamma$ can be found in [3]. One important point to note here is that these filters are scale-dependent. We have assumed mirror boundary conditions in our implementation. We have set $\mu=c s t$ for all wavelet levels, and the scaling function coefficients were left unchanged. The degree of smoothness in the spatial domain compared to the temporal domain can be tuned by considering different depths $J_{s}$ and $J_{t}$ of the wavelet decomposition.

To avoid the typical blocky artefacts when using the orthogonal DWT we have used the wavelet cycle-spinning, as proposed in [8]. This method can be applied in the current setting and tries to achieve some level of translation invariance by choosing a randomly shifted DWT at each iteration of (8).

\section{F. Experimental results}

1) $1 D$ evaluation of the E-spline wavelets: To illustrate the possibilities of the E-spline wavelets in dynamic PET reconstruction, we performed a curve-fitting experiment on a blood pool time activity curve encountered in dynamic ${ }^{13} \mathrm{~N}$ ammonia PET [9]. The time activity curve was given by an exponential polynomial [9] corrupted by zero-mean Gaussian noise of various variance $\sigma^{2}$

$$
y(\tau)=(\tau)^{2} \exp (-0.4 \tau)+c s t+\mathcal{N}(0, \sigma),
$$

and was sampled at times $0.5,1.5, \ldots, 99.5$. The variance was set to achieve data SNR of $0 \mathrm{~dB}$ and $20 \mathrm{~dB}$ respectively.

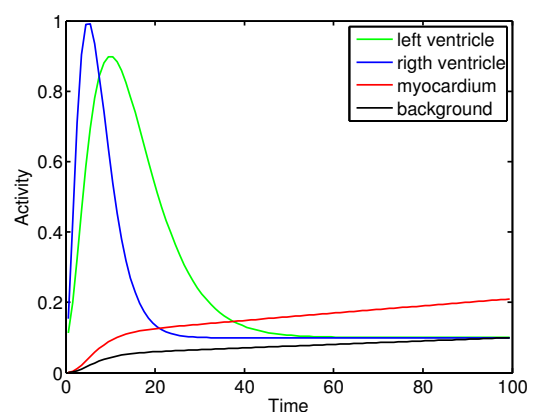

(a)

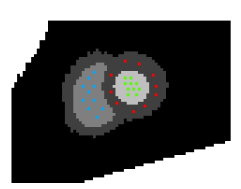

(b)
Fig. 1. The tomographic simulation experiment. (a) TACs for the different regions and (b) slice of the NCAT phantom with the positions of the voxels used for the analysis (colour coded).

We evaluated the SNR of the curves reconstructed from the largest $n$ wavelet and scaling coefficients ( $\ell_{0}$-norm). The wavelet decomposition consisted of 5 levels and results were averaged over all possible shifts with 100 realizations per shift. We used orthogonal E-spline wavelets with $\boldsymbol{\alpha}=$ $(0,-\alpha,-\alpha,-\alpha)$ where $\alpha$ ranged from 0 to 1 in steps of 0.05 .

2) Tomographic simulation study: We considered 2+1D (spatial + temporal) simulations. The phantom consisted of 100 time frames of $100 \times 80$ pixels. The phantom was a slice of the NCAT phantom containing the myocardium [10]. Four different TACs were simulated for respectively the left and right ventricle, the myocardium and the background. The time curves in the ventricles were given by exponential polynomials [9], the time curves of the myocardium was derived from the left ventricular input function using a two compartmental model [9]. The phantom and the time curves are illustrated in Fig. 1.

The simulated detector was set up as a geometric approximation of a commercial scanner consisting of 616 detector crystals in a ring. Photons were emitted back-toback according to an inhomogeneous Poisson process.

We simulated 10 noise realizations of the phantom. Each realization consisted of almost 800,000 detected pairs. The reconstructions were obtained after 100 iterations of (8) with $\lambda^{0}$ equal to zero. The projector for the reconstruction $\mathrm{P}$ was based on the line-length model and involved multiple tracings per detector pair. The reconstructed SNR was evaluated at 30 pixel locations, 10 per representative region (left and right ventricle and left myocardium, see Fig. 1). Thus, for the three regions we had 100 reconstructed TACs. We calculated the SNR and the corresponding 95\% confidence interval using the bootstrap method. Results were obtained for different tuning parameters $\mu$.

The temporal depth of the orthogonal wavelet decomposition was set to $J_{t}=5$, while the number of spatial levels $J_{s}$ was varied from 0 to 2. Two different $\boldsymbol{\alpha}$ 's were considered. The conventional (polynomial) B-spline wavelets using $\boldsymbol{\alpha}=(0,0,0,0)$, corresponding to $4^{\text {th }}$ order Battle-Lemarié wavelets and E-spline wavelets with $\alpha=$ $(0,-0.6,-0.6,-0.6)$. 


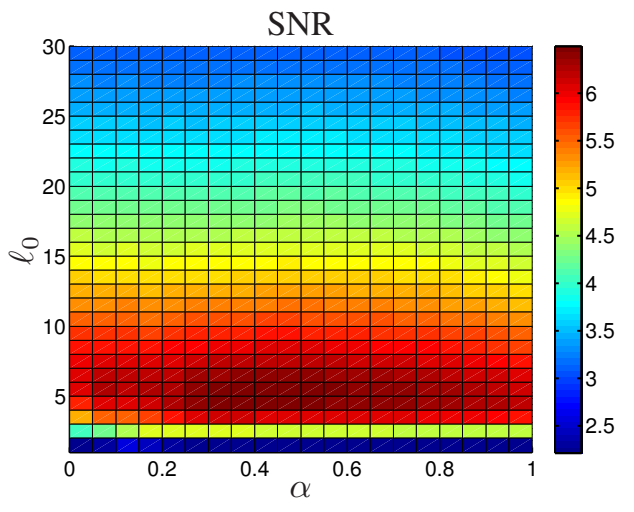

(a)

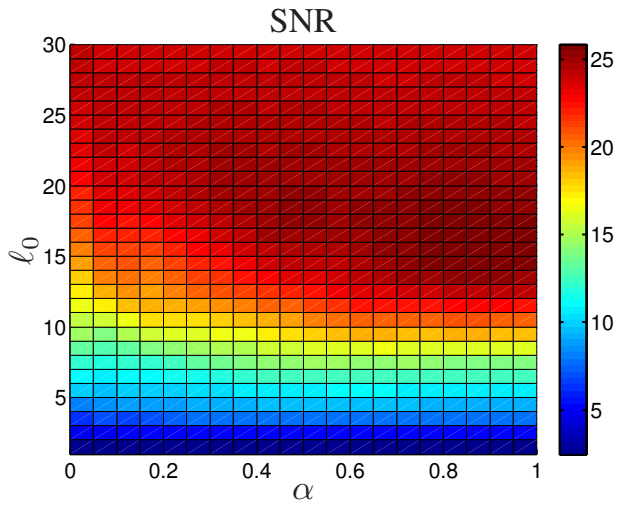

(b)

Fig. 2. SNR as a function of the number of non-zero coefficients and $\boldsymbol{\alpha}$ for the curve fitting example. (a) input SNR $=0 \mathrm{~dB}$ and (b) input SNR $=$ $20 \mathrm{~dB}$.

\section{RESULTS}

\section{A. $1 D$ evaluation of the E-spline wavelets}

The results for the 1D study are shown in Fig. 2. The performance of the conventional B-spline wavelets corresponds to $\alpha=0$. For high noise levels (close to $0 \mathrm{~dB}$ ), the best results are obtained for a low number of coefficients (less than 10). The best performance is obtained for $\alpha=0.5$ (note again that $\alpha=(0,-\alpha,-\alpha,-\alpha)$ ) and is about $1 \mathrm{~dB}$ better than the results for the B-spline wavelets.

A higher number of coefficients can be used for the medium noise range (around $20 \mathrm{~dB}$ ). For $\alpha>0$ the best results are obtained for a smaller number of coefficients as compared to the conventional B-spline wavelets. For $\alpha=0.6$, the best results are already obtained using only 15 coefficients whereas about 25 coefficients are necessary for the B-spline wavelets. This illustrates that a sparser representation is possible when using the appropriate Espline wavelets. Moreover, the best performance for $\alpha=0.6$ is about $3 \mathrm{~dB}$ better than for the best performance using $\alpha=0$. Finally, from Fig. 2 it can be seen that there is a broad range of $\alpha$ 's that can produce sparser representations and perform better than the conventional B-splines.

\section{B. Tomographic simulation study}

The results for the tomographic reconstruction are shown in Fig. 3. The highest reconstructed SNR is always found when using the E-spline wavelets. A significant difference (no overlapping $95 \%$ confidence intervals) was found for the right and left ventricular time curves for using respectively $0-2$ and $0-1$ spatial decomposition levels. The SNR is improved for a higher number of spatial decompositions, however note that this reduces the spatial resolution. This is not reflected in the current SNR curves because the regions where we sampled the time curves were kept away from the borders.

Reconstructed slices using different spatial levels of decompositions are compared to the non regularized solution in Fig. 4.

\section{CONCLUSION}

We have demonstrated the beneficial use of E-spline wavelets in combination with $\ell_{1}$ spatio-temporal regularization in dynamic PET imaging. The E-splines were found to be advantageous over conventional polynomial B-spline wavelets in modelling TACs.

\section{REFERENCES}

[1] I. Daubechies, M. Defrise, and C. D. Mol, "An iterative thresholding algorithm for linear inverse problems with a sparsity constraint," Commun. Pure Appl. Math., vol. 57, no. 11, pp. 1413-1457, 2004.

[2] R. Lewitt and S. Matej, "Overview of methods for image reconstruction from projections in emission computed tomography," Proc. IEEE, vol. 91, no. 10, pp. 1588-1611, 2003.

[3] I. Khalidov and M. Unser, "From differential equations to the construction of new wavelet-like bases," IEEE Trans. Signal Process., vol. 54, no. 4, pp. 1256-1267, April 2006.

[4] S. Mallat, "A theory for multiresolution signal decomposition: the wavelet representation," IEEE Trans. Pattern Anal. Mach. Intell., vol. 11, no. 7, pp. 674-693, 1989.

[5] M. Unser and T. Blu, "Wavelet theory demystified," IEEE Trans. Signal Process., vol. 51, no. 2, pp. 470-483, Feb. 2003.

[6] — "Cardinal exponential splines: part I - theory and filtering algorithms," IEEE Trans. Signal Process, vol. 53, no. 4, pp. 14251438, Apr 2005.

[7] M. Unser, "Cardinal exponential splines: part II - think analog, act digital," IEEE Trans. Signal Process., vol. 53, no. 4, pp. 1439-1449, Apr 2005

[8] M. Figueiredo and R. Nowak, "An EM algorithm for wavelet-based image restoration," IEEE Trans. Image Process., vol. 12, no. 8, pp. 906-916, Aug. 2003.

[9] S. R. Golish, J. D. Hove, H. R. Schelbert, and S. S. Gambhir, "A fast nonlinear method for parametric imaging of myocardial perfusion by dynamic N-13-ammonia PET," J. Nucl. Med., vol. 42, no. 6, pp. 924-931, 2001.

[10] W. Segars, D. Lalush, and B. Tsui, "A realistic spline-based dynamic heart phantom," IEEE Trans. Nucl. Sci., vol. 46, no. 3, pp. 503-506, 1999. 
$J_{s}=0$

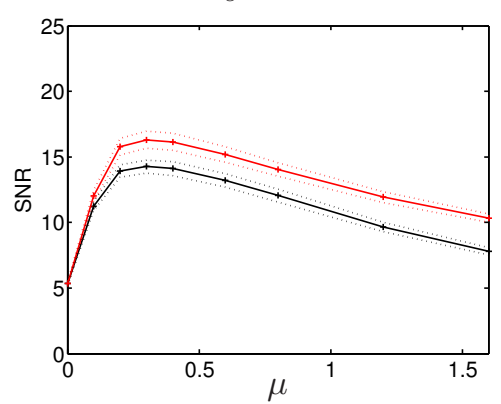

LV
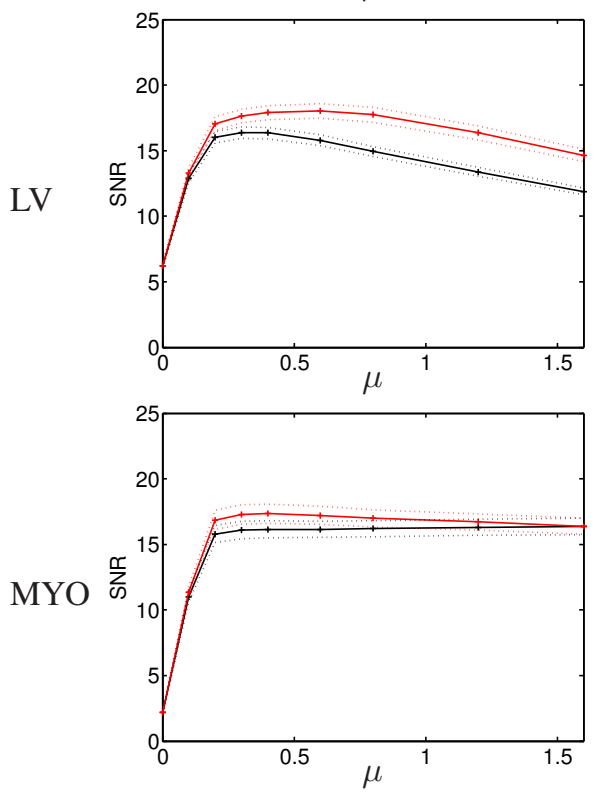

$J_{s}=1$
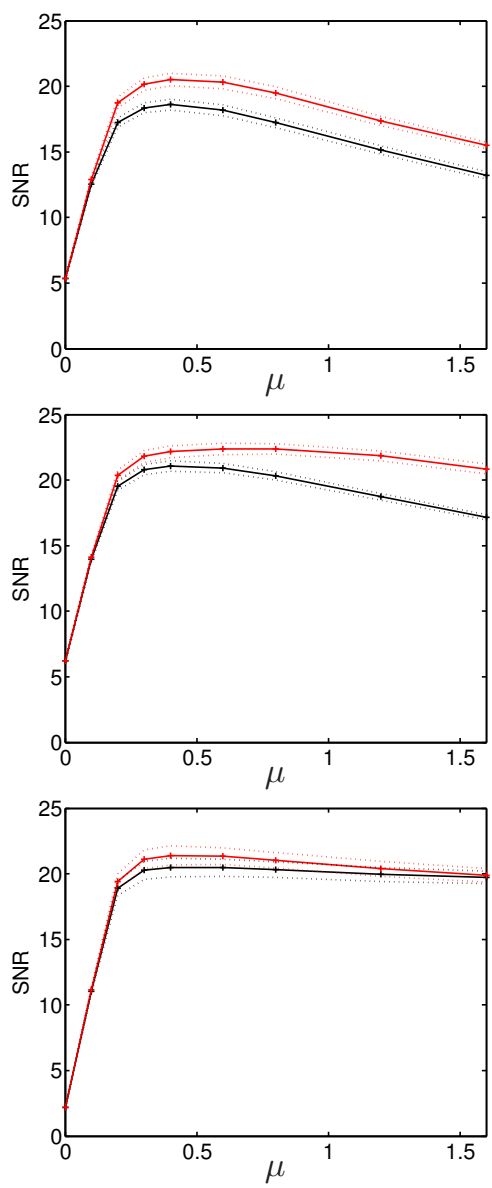

$J_{s}=2$
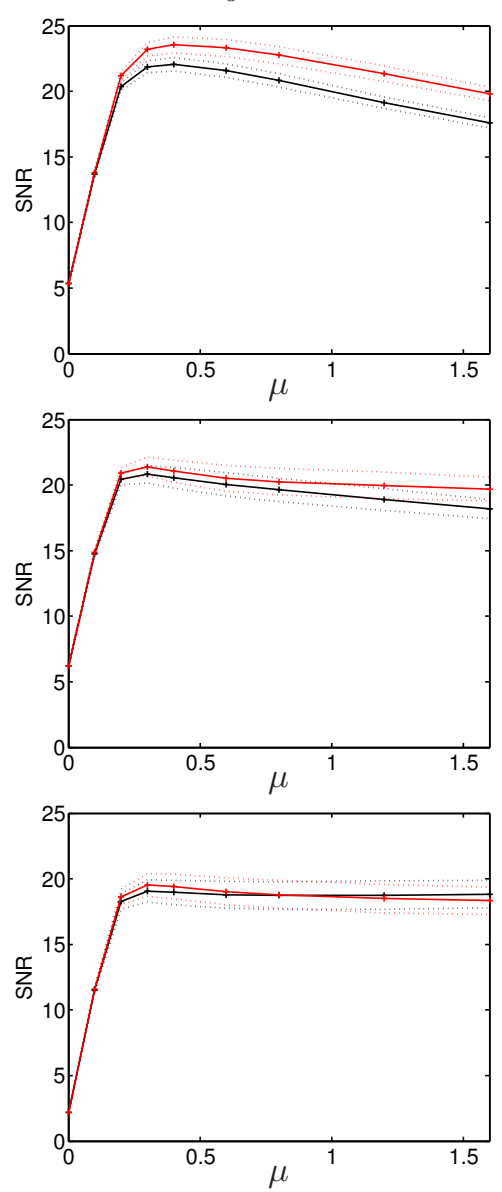

Fig. 3. SNR as a function of $\mu$ for the three regions and for a different number of spatial decomposition levels for the tomographic reconstruction example. (-) SNR obtained using the conventional polynomial B-spline wavelets $(\boldsymbol{\alpha}=(0,0,0,0))$ and $(\cdots \cdots)$ the corresponding $95 \%$ confidence interval. ( - ) SNR obtained using E-spline wavelets $(\boldsymbol{\alpha}=(0,-0.6,-0.6,-0.6))$ and $(\cdots)$ ) the corresponding $95 \%$ confidence interval. It can be observed that E-splines consitently outperform regular wavelets.

$$
\mu=0
$$
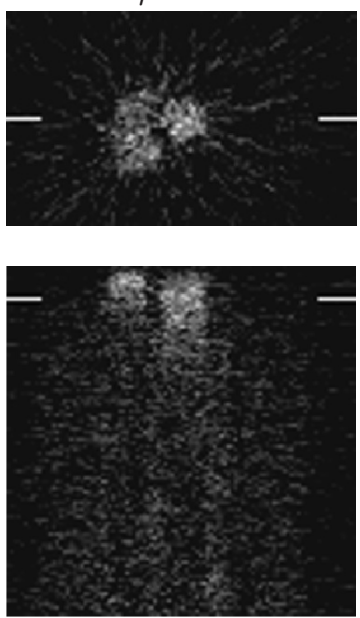

$$
J_{s}=0
$$

$\mu=0.4$
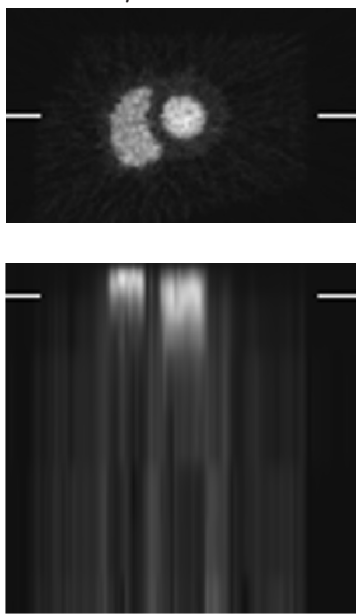

$J_{s}=1$

$\mu=0.4$
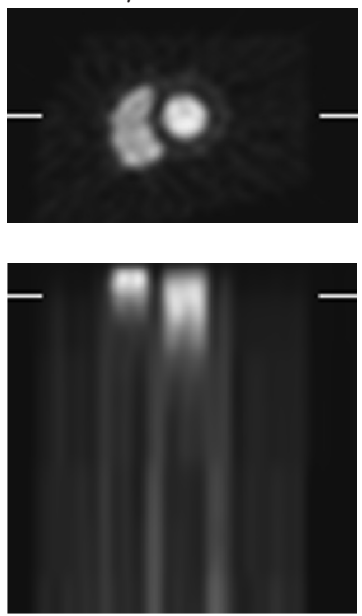

$J_{s}=2$

$\mu=0.4$
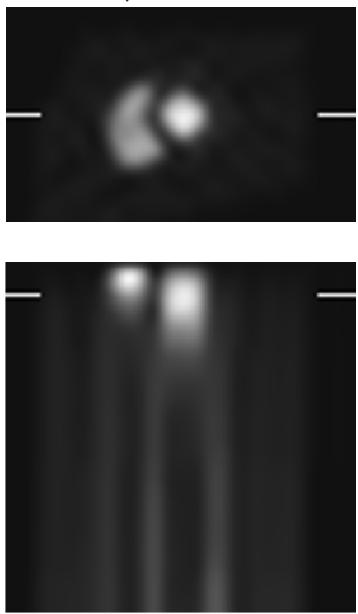

Fig. 4. Reconstructed slices using different number of spatial decomposition levels and tuning parameter $\mu$. Top and bottom row are spatial and temporal slices, respectively. The time and space locations are indicated by the white bars. The results in the third column give a good compromize between spatial and temporal regularization. 